\title{
Ubber die psychiatrische Bedeutung der Erkrankungen der subcorticalen Ganglien und ihre Beziehungen zur Katatonie.
}

\author{
Von \\ Dr. Fritz Fränkel (Berlin).
}

(Aus dem Bürgerspital Stuttgart [Chefarzt: Geh.-Rat Dr. Fauser].)

(Eingegangen am 29. Mai 1921.)

„Es gibt keinen Umstand, der so sehr beweist, wie weit bisher noch unsere Beobachtungsfähigkeit zurück ist, als der Umstand, daß wir bis heute weder von den Funktionen des Corpus striatum noch von den Symptomen etwas wissen, die eintreten, wenn es zerstört oder wenn es gereizt ist." In demselben Jahr, in dem Ludwig Edinger ${ }^{10}$ ) diese Worte schrieb, erschien die berühmte Arbeit Wilso $\mathrm{ns}^{44}$ ) über die progressive lentikuläre Degeneration, und die umfangreiche neurologische Forschung, die sich an sie anschloB, hat unsere himphysiologischen Kenntnisse bedeutend erweitert und die große Bedeutung, die Edinger den subcorticalen Ganglienmassen zuspricht, bestätigt. Aber wie so oft hat vertieftes Eindringen nicht nur die Erkenntnis, sondern auch die Problematik erhöht, und die Probleme, die sich an die Untersuchungen der Basalganglien anschließen, berühren nicht nur die Neurologie, sondern sie sind auch für spezielle Fragen der Psychiatrie von großer Wichtigkeit. Wenn Edinger (l. e.) z. B. ausspricht, es sei ihm nicht unwahrscheinlich, daß ein guter Teil von dem, was man kurz als Spontaneität der Bewegung bezeichnet, durch das Striatum vermittelt werde, so ist damit schon angedeutet, da $B$ gerade ein psychischer Anteil des Bewegungsvorganges, eben der Antrieb, in funktionale Beziehung zum C. striatum gesetzt wird. Und so ergibt sich für den Psychiater eine doppelte Fragestellung: Welche psychischen Störungen finden sich bei Erkrankungen der subcorticalen Ganglien und weiter, bei welchen Geisteskrankheiten finden sich körperliche Symptome, die auf jene als Sitz hinweisen?

Ohne dem historischen Gang der Pathographie in den Einzelheiten zu folgen, sei an die Krankheitsgruppe aus der Reihe der extrapyramidalen Störungen angeknüpft, die heute im Mittelpunkt des neurologischen Interesses steht, die Pseudosklerose bzw. die Wilsonsche Krankheit. Das Leiden macht sich bekanntlich geltend in einer erheblichen Störung des Motoriums, die einerseits zu einer allgemeinen 
Bewegungsarmut (Fixationsrigiditat, mimische Starre) führt, andererseits mit einer mitunter sehr erheblichen Hyperkinese verbunden ist, deren Zeichen ein der Schüttellähmung ähnelnder Tremor oder athetotische Erscheinungen sein können. Daneben bestehen als häufiger Befund schwere Sprach- und Schluckstörungen, Alterationen des Vasomotoriums und nicht selten Blasen-, manchmal auch Mastdarminkontinenz. Die Krankheit verläuft oft schubweise, Anfälle epileptischer Art sind wiederholt beobachtet; auch Pupillenstörungen werden von einzelnen Autoren angegeben. Im Krankheitsverlauf kommt es zur Ausbildung von Contracturen (Pfötchenstellungen der Finger u. ä.). Trotz des progressiven Charakters kann sich das Leiden viele Jahre hinziehen. Wie wir seit Wilsons Monographie wissen, ist von den inneren Organen die Leber miterkrankt. Welche Erklärungen für diese Beteiligung möglich sind, soll ebensowenig wie andere der noch sehr strittigen Einzelheiten des Krankheitsbildes hier erörtert werden. Uns interessiert vielmehr die Veränderung der Psyche, die bei den Kranken fast nie vermißt wird. Leider vernachlässigt die große Kasuistik, die heute über die Pseudosklerose besteht, den psychischen Faktor sehr, und es dürfte kein Zufall sein, daß vielleicht der ausführlichste und für die psychiatrische Beurteilung genauest beschriebene Fall von dem Entdecker der Krankheit, von C. Westphal ${ }^{42}$ ) herrührt. Im Vordergrund der seelischen Störungen steht bei seinem Kranken (Fall 2) eine Affektreizung. Der früher ganz normale Kranke bekam Wutanfälle, bei denen er laut schrie und brüllte, mitunter auch gewalttätig wurde, so daß er isoliert werden mußte; daneben finden sich bei ihm traumhafte Zustände, in denen er keine Nahrung zu sich nimmt, unreinlich ist und nach deren Abklingen er über eigenartige Sensationen klagt: er habe so ein putziges Gefühl gehabt, es sei ihm gewesen, als ob ihm die Knochen entzweigebrochen wären. In der weiteren Beobachtung wechselte Besserung und Verschlimmerung des Zustandes; es kam mitunter zu Illusionen und Halluzinationen; die Reizbarkeit nahm einen chronischen Charakter an. Ein Jahr vor dem Tode des Kranken verzeichnet die Krankengeschichte wieder Anfälle äußerster Wut, größte Reizbarkeit, zum Teil sicher auf Grund von Halluzinationen oder Gehörsillusionen; zu anderen Zeiten ist er friedlich-heiterer Stimmung, wobei er ein gutes Gedächtnis und richtiges Urteil über Zeit und Ort aufwies. Soweit in großen Zügen wiedergegeben West phals Schilderung. In dem ersten Fall, der in der gleichen Arbeit veröffentlicht ist, sind die seelischen Erscheinungen weniger auffällig, aber die Beobachtungen des Autors sind doch sehr wichtig. Es handelt sich bei dem an Pseudosklerose erkrankten Mann um eine intellektuelle Schwäche ohne Sinnestäuschungen und Wahnideen. Aber wie Westphal schreibt ,zeigte diese intellektuelle Schwäche gewisse Eigentümlichkeiten, bei auf- 
fallendem Mangel aller Spontaneität und gleichbleibender Indifferenz der Gemütslage war die Auffassung und das Verständnis relativ gut; doch bewahrte das Gedächtnis für gewisse ihm gegenüber gebrauchte, übrigens ganz gleichgültige Phrasen eine merkwürdig lange, Wochen, ja Monate dauernde Erinnerung, und es machte oft den Eindruck, als habe der Patient, seitdem er die betreffende Phrase gehört, in der Zwischenzeit nichts anderes gedacht und knüpfe nun wieder daran an. Eine Art Beharrungsvermögen für gewisse Gedanken, ähnlich dem Beharrungsvermögen der Aphasischen".

Es dürfte nicht zu bestreiten sein, daß, wenn man diese Fälle unter die gewöhnlichen Kategorien subsumieren wollte, am ehesten die Dementia praecox in Betracht käme. In der Tat erinnern auch die in psychiatrischer Beziehung weniger gut beschriebenen Fälle anderer Autoren - vor allem hat sich v. Strümpell ${ }^{37}$ ) um die Ausgestaltung des Krankheitsbildes verdient gemacht*) -, aber auch FranklHochwarts ${ }^{15}$ ) Fall u.a., am meisten an katatone Zustände. Besonders deutlich ist dies bei den Kranken Fleischers $\mathrm{s}^{13}$ ), der als erster die eigentümliche Verfärbung der Hornhaut bei Pseudosklerose beschrieb. Einmal handelt es sich um einen 23jährigen Bauernsohn, der bei der ersten Aufnahme melancholisch-weichmütiger Stimmung ist, bei der zweiten auch zunächst depressiv, dann aber stuporös wird, zuweilen explosiv schimpft und Verfolgungsideen äußert. Noch deutlicher ist die Beziehung in einem anderen seiner Fälle. Ein 28jähriger Kaufmann mit anfallsweisen Erregungszuständen, bei denen er seine Frau totzustechen droht, dabei geistig stumpf, sexuell sehr erregt ist. In der Anstalt (Göppingen) ist er von heiter-läppischem Wesen, aber auch renitent und barsch, später teilnahmlos und interesselos, durch den Tod seines Kindes kaum bewegt; mitunter Erregungszustände, in denen er auf den Arzt schimpft und spuckt, öfters unanständiges Benehmen, steht auf den Kopf, gibt einem Patienten eine Ohrfeige, geht zu anderen und läßt einen Flatus, zeigt seine Natur, ist daneben über seine Lage orientiert, rechnet gut. Plötzlicher Exitus. Sektion ergibt Hyperämie der Hirnhäute. - Die Pseudosklerose galt bekanntlich bis Wilsons Publikation für funktionell. Als einzigen anatomisch wertvollen Befund verzeichnet Frankl-Hochwart in seiner $\mathrm{Zu}$ sammenstellung (l. c.) in 2 Fällen eine auffallende Härte der Hirnkonsistenz, ein Befund, auf dessen Bedeutung noch zurückzukommen sein wird. - Er erwähnt im übrigen, daß in $80 \%$ aller Fälle psychische

*) In psychischer Hinsicht sind seine Fälle ebenfalls ausgezeichnet durch sehr starke affektive Erregbarkeit, die in einem Fall zu Zorn und Wutanfällen, im anderen zu Heiterkeitsausbrüchen, Zwangslachen und Weinen führt. In dem fortgeschritteneren Verlauf werden Apathie und Stumpfheit, Schlafsucht, einmal Verblödung, ein anderes Mal Halluzinationen notiert. 
Störungen vorhanden sind, besonders Reizbarkeit bis zur Tobsucht auf der einen, Apathie auf der anderen Seite, er selbst beobachtet barockes Wesen in seinem Fall mit großer Weitschweifigkeit und anderen Abnormitäten.

Aus der jüngeren Literatur sei noch folgendes kurz erwähnt. Wilso $^{11}$ ) selbst, der auf die psychischen Symptome nur kurz eingeht, aber vor ihrer Unterschätzung warnt, sieht die Eigenart der seelischen Veränderungen in einer gewissen Fügsamkeit, Leichtigkeit, Kindlichkeit, Euphorie; daneben erhöhte Reizbarkeit, Zunahme der gemütlichen Reaktionen, besonders Zwangslachen, jedoch keine Demenz wie bei der Paralyse oder dem Senium, auch gegen die Dem. praecox. sei der Unterschied deutlich, doch bestehe eine gewisse Einengung des seelischen Horizontes. Daß eine Verblödung*) nicht ausgeschlossen ist, beweisen mehrere Fälle, so der von $\mathrm{Maas}^{27}$ ), bei dem mit 50 Jahren eine völlige Demenz vorhanden ist. Doch schon vorher versagte der Kranke bei den leichtesten Rechenaufgaben, war völlig teilnahmlos. Auch die Abgrenzung gegen die Katatonie kann unter Umständen recht schwierig sein. So beschreibt Kastan ${ }^{21}$ ) einen Soldaten, bei dem während 8 Jahren der Krankheitsverlauf verfolgt werden konnte. Bei intakter Intelligenz, guter Merkfähigkeit bestand ein eigentümliches, als kataton gedeutetes Wesen, Sinnestäuschungen, Wahnbildungen, Zornaffektausbrüche, Grimassieren, Stereotypien. Ferner aber die in Schüben auftretenden Zeichen einer Pseudosklerose (Maskengesicht, Speichelfluß usw.),

Ein ähnliches Mischbild beschreibt A. Westphal41); ein junges Mädchen, das einerseits eine Reihe amyostatischer Symptome, Bewegungsarmut, Fixationsrigidität, vermehrte Muskelspannung, doppelte Athetose, andererseits ein läppisches, stuporöses Verhalten mit Manieren, Wortneubildungen, Wahnideen, Trugwahrnehmungen aufwies. Westphal erörtert kurz die Beziehungen der körperlichen zu den psychischen Erscheinungen, die des Stupors zur Bewegungsarmut, des "Schnauzkrampfes" zu den Muskelspasmen. Wir kommen darauf noch zurück.

Jedenfalls ist bei der verhältnismäßigen Seltenheit der hier in Betracht kommenden Krankheitsbilder und der ziemlich kurzen Zeit ihrer genaueren Analyse die Kombination mit so ausgesprochen katatonen Zügen doch sehr auffällig und sollte kein Zufall sein.

*) Nach Hoes s slin ${ }^{18}$ ) führt die eine Form der geistigen Störungen bei Pseudosklerose geradezu von einfachen Schwächezuständen, gemütlicher Stumpfheit, Abnahme der intellektuellen Fähigkeiten langsam zur Demenz, bei der anderen kommt es zu akuten Störungen, plötzlichen Charakterveränderungen, Reizbarkeit, Heftigkeit, Zerstörungswut, Anfällen von Sinnestäuschungen und Verwirrtheitszuständen. Mir scheinen die Verlaufsarten nicht scharf getrennt zu sein. 


\section{F. Fränkel: Über die psychiatrische Bedeutung der Erkrankungen}

Aus eignen Beobachtungen ${ }^{17}$ ) seien folgende Momente erwähnt:

Bei einem im Anschluß an ein Trauma Erkrankten entwickelten sich die seelischen Veränderungen durchaus parallel mit den körperlichen. Die aufs äußerste gesteigerte gemütliche Reizbarkeit des Kranken, die Beschränkung seiner Interessen auf das eigene Ich sind prae morbo auch nicht angedeutet vorhanden. Die oft hervorgehobene Beziehung der Basalganglien zur Affektivität hat hier quasi experimentell eine Bestätigung gefunden. Besonders möchte ich hervorheben das Ergebnis von Assoziationsversuchen, die ich im Beginn der Krankheit an einem zur militärischen Begutachtung in das unter der Leitung von Geheimrat Fauser stehende Lazarett Eingewiesenen machen konnte. Es handelt sich um einen früher stets gesunden, von zuverlässiger Seite als lebhaft und intelligent geschilderten Mann, bei dem in französischer Gefangenschaft die ersten Erscheinungen der Krankheit in Form von Gehstörungen auftraten. Zur Zeit bestanden bei völligem Fehlen von Pyramidenzeichen ein leichter Spannungszustand der Muskulatur, auffallende mimische Starre, vereinzelte Zitterbewegungen der Hände, athethotische Zehenbewegungen, allgemeine Bewegungsarmut. Ausgesprochene Neigung zu Zwangslachen, das als besonders unangenehm empfunden wird, ohne daß dem Kranken das Zwangsmäßige der Reaktion klar ist, er beklagt sich nur, daß er so leicht lachen müsse, findet aber immer Begründungen für den einzelnen Ausbruch.

Die Assoziationsprüfung ergab nun, daß bei durchaus normaler Reaktionszeit und obwohl man keineswegs von einer Verarmung der durch das Reizwort ekphorierten Vorstellungsreihen sprechen kann, einzelne beweisen sogar ein gewisses intellektuelles Niveau, das über den Durchschnitt ragt (Elend-Wohnung, Mord - rätselhaft usw.) doch die Neigung zur Wiederholung der gleichen Reaktionen besteht, die innerhalb der ersten 10 Reizwörter dreimal ihn ,Baum“ antworten läßt, der nächsten 20 dreimal Mann und gegen Ende des Versuches auf jedes Eigenschaftswort die Antwort, ,sehr" (zehnmal) und auf andere Worte achtmal ,groß" geben läßt, so daß von den letzten 50 Antworten zwei Gruppen von acht und zehn Antworten einfach stereotyp sind. Ich möchte diese psychische Rigidität mit der Neigung zu der körperlichen Beharrungstendenz in Beziehung setzen und erinnere wieder an den erst geschilderten Kranken von Westphal (ähnlich wird bei Bleuler") die ,Affektsteifigkeit" der Schizophrenen hervorgehoben). Übrigens hatte unser Patient subjektiv durchaus keine Empfindung einer Denkhemmung, meinte sogar, es drängen sich ihm mehr Gedanken auf als früher. Bei Befragung erzählt er aber, es sei immer wieder dasselbe, er müsse soviel an die Kriegserlebnisse denken, was früher nicht der Fall war. Also auch hier eine gewisse Steifigkeit intellektueller Art. 
In einem weiter vorgeschrittenen Fall*) dagegen war eine sehr starke Verlängerung der Reaktionszeit zu konstatieren, außerdem erfolgte unter 40 Reizworten achtmal überhaupt keine Antwort mit der Begründung, mir fällt nichts ein. Auch hier waren die Reaktionen ohne eine Komponente von intellektuellen Defekten.

Daß die Analogie mit der Perseveration des Aphasikers auch bei Westphal nur ein Bild ist, daß die hier vorliegende Art der Störung eine prinzipiell andere ist, braucht nicht ausgeführt zu werden. Es handelt sich um keinen Ausfall des assoziativen Erfahrungsschatzes, sondern um eine Erschwerung seiner Ansprechbarkeit. Zwingt man den ersterwähnten Kranken zu einer größeren Aufwendung seelischer Arbeit für die gestellte Aufgabe, gelingt es auch, andere Reaktionen zu erhalten. Da der Versuch eine gewisse Einstellung verlangt, da es sich nicht um ein bloßes Hingeben auf den akustischen Eindruck handelt, sondern eine aktive Anspannung erforderlich ist, wird der Ausfall nicht nur die inhaltliche Seite (etwaige Komplexe, geistige Dürftigkeit usw.) aufdecken, er wird auch einen $\operatorname{Schlu} B$ auf das Maß der vorhandenen geistigen Energie gestatten, und daß hier sowohl die Häufigkeit der Wiederholung wie die Verlängerung der Reaktionszeit bzw. das Ausbleiben der Reaktion nur im Sinne einer herabgesetzten aktiven psychischen Energie angesehen werden kann, erscheint mir gewiß. Sonst wäre, da möglicherweise affektbetonte Reizworte wie „Rente" in dem ersten „Tod“ u. a., in dem zweiten Fall keinerlei Einfluß hatten, was sich auch in der Analyse bestätigte, die Ungleichmäßigkeit der Leistung ganz unverständlich, das Nebeneinander von reichen, sogar nicht ganz durchschnittlichen Leistungen mit dürftigen, bequemen oder die Anstrengung ganz vermeidenden. Ich denke, man wird bei größerem Material eine ganze Skala feiner Abstufungen der verminderten psychischen Aktivität in ihrer Manifestation im Assoziationsexperiment aufstellen können.

Zusammenfassend kann gesagt werden: Die Alteration der Psyche gehört zu dem Bilde der Pseudosklerose und der Wilsonschen Krankheit. Unter welchen Bedingungen sie vermißt wird, kann heute noch nicht erklärt werden, keineswegs ist, wie genaue Untersuchungen neuerer Zeit beweisen, das Übergreifen des Krankheitsprozesses auf die Rinde die Ursache der seelischen Ausfälle, denn in vielen Fällen (so Economo $0^{9}$ ), Maas, l.c.) kann eine Beteiligung der Rinde ausge-

*) Ich verdanke die Gelegenheit zur Untersuchung Herrn Boenheim, dem ich hierfür auch an dieser Stelle meinen Dank sage. Er hatte auch die Freundlichkeit, mir den von ihm jüngst publizierten Fall von jugendlicher Pseudosklerose zu demonstrieren, bei dem eine Art Schnauzkrampf zunächst den Verdacht der Katatonie erweckt hatte. 
schlossen werden. Vielleicht ist die Dauer der Krankheit hierfür entscheidend*).

Die Art der Geistesstörung ist nicht einheitlich, es kommt zu tobsuchtsartigen Erregungszuständen wie zu Depressionen, zu Verblödungen wie Intaktbleiben der Intelligenz und des Gedächtnisses. Jedoch kann man feststellen, daß die häufigste Form der ,symptomatischen Psychose" durchaus an katatone Zustandsbilder teils voll ausgeprägt, teils in forme fruste erinnert. Ausgeprägter als Sinnestäuschungen und Wahnbildungen ist hier von den Symptomen einer Herabsetzung der psychischen Aktivität [im Sinne Berzes $\left.{ }^{31}\right)$ ] deren Manifestation als Einengung des seelischen Horizontes (Wilson), die affektive Reizbarkeit•bzw. die Stumpfheit und Apathie, bis zum Stupor gesteigert, und schließlich die Herabsetzung der intellektuellen Leistung, wie sie sich etwa in den angeführten Assoziationsversuchen bemerkbar macht, als die Erschwerung einer Intensivierung der intellektuellen Arbeit, daher Haftenbleiben an gefaßten Reaktionen, trotz der potientiellen Fähigkeit zur Volleistung. Ein Wort noch zu der Affektstörung. Daß es sich bei den oft erwähnten Affektausbrüchen der Kranken nicht einfach um psychopathische Reaktionen handelt, beweist das Vorhandensein der gemütlichen Zwangserscheinungen, Zwangslachen und Weinen. Dieses eigenartige Phänomen, das wohl noch nicht hinreichend psychologisch erfaßt worden ist, scheint mir gewissermaßen das Kriterium für eine ,organische“ Herabsetzung der Reizschwelle gegen gemütliche Reize zu sein und so die Unterscheidung gegen die (psychogene) affektive Reizbarkeit der Psychopathien zu gestatten. Zugleich bedeutet aber die Unfähigkeit, seelische Reize affektlos zu beantworten, daß der Willensapparat oder besser die psychische Aktivität, deren Aufgabe auch die Überwindung der affektiven Tendenz unserer seelischen Mechanismen ist, geschwächt ist. Man kann nicht einwenden, daß es sich bei dem Zwangslachen und Weinen um rein körperliche Vorgänge handelt, denn obwohl die Kranken unserer Beobachtung selbst bemerken, daß sie leichter lachen oder weinen als in gesunden Tagen, empfinden sie die einzelne Reaktion durchaus nicht als zwangsmäßig, sondern sind stets imstande, sie intellektuell zu begründen, empfinden irgendeine Kleinigkeit als komisch, selbst wenn die Gesamtaffektlage durchaus nicht zum Frohsinn geneigt ist.

Was die anderen Typen der Erkrankungen der Basalganglien betrifft, so sei, abgesehen von der Betrachtung der Parkisonschen Schüttellähmung, deren enge Beziehungen zur Pseudosklerose wohl sichergestellt, deren anatomisches Substrat jedoch noch nicht widerspruchslos

*) In Hoesslins Fall wies Alzheimer eine Beteiligung der Rinde nach. Diese ist, wie auch in Spielme yers $\mathrm{s}^{35}$ ) Untersuchungen, diffus erkrankt, ohne Bevorzugung bestimmter, lokalisatorisch ausgezeichneter Regionen. 
erfaßt zu sein scheint. Auch tritt die Schüttellähmung meist in hohem Alter auf, so daß die bei ihr ja fast stets vorhandenen seelischen Veränderungen von den Altersprozessen schwer zu trennen sind, die Kombination der Paralysis agitans mit Dem. praec. ist einmal beschrieben (Frommer).

Aber eine Abart derselben, die Paralysis agit. sine agitatione, hat jüngst ihre lokalisatorische Erfassung gefunden durch einen interessanten, auch psychiatrisch bedeutungsvollen Fall. H.Deutsch ${ }^{8}$ ) berichtet von einer 36 jährigen Patientin, daß sie von einem Hausknecht überfallen, mit der Hand und mit einem Strick gewürgt wurde, 5 Tage danach Krämpfe; nach den Anfällen blieb eine Lähmung der rechten Hand und des rechten Beines, ferner bestand eine Starre der Muskulatur, das Gesicht war ausdruckslos, die Lippen nach unten gezogen; es entwickelten sich allmählich Contracturen an den Knien und Füßen. Die Kranke wurde völlig apathisch, feste Bissen hielt sie oft stundenlang im Munde, ohne zu schlucken. Deutsch bezeichnet ihren Zustand als halb stuporös und fügt hinzu, es ließe sich nicht auseinanderhalten, wieviel von diesem Verhalten allgemein psychischen Veränderungen, wieviel den beschriebenen Innervationsstörungen zuzuschreiben ist. Erwähnt sei noch die langsame schleppende Sprache, die flüsternd und mühsam war. Exitus nach 3 Monaten ergab nur eine symmetrische Erweichung der Linsen- und Schwanzkerne. Einen ähnlichen Fall, allerdings ohne Befund am Gehirn (keine mikroskopische Untersuchung) erwähnt Bause ${ }^{29}$ ) 1914. Ein 19jähriger Lehrling erhängt sich aus Spielerei, er wird nach 10 Minuten abgeschnitten, $3^{1 / 2}$ Stunden bewußtlos, dann schwere Jactationen, es bildet sich ein Zustand aus, in dem Apathie, Apraxie (motorische?), Spasmen, cerebellare Ataxie bemerkt wurden sowie eine Pupillenstörung. Späteres Verhalten stumpf und abweisend, Tod nach 7 Monaten an Miliartbc. Und schließlich sei auf die Arbeit von E. Meyer ${ }^{29}$ ) über psychische Störungen nach Strangulation in diesem Zusammenhang hingewiesen. In ihr berichtet er, daß die seelischen Ausfallserscheinungen vor allem in einer Gedächtnisstörung nach Art des Korsak off bestanden. Interessanterweise beobachtete er nun bei dem Strangulierten, daß die Sprache zitternd, stotternd, verschwommen war; ferner bestand eine Neigung, Stellungen festzuhalten. Auch wird erwähnt, daß Verlegenheitsbewegungen, die an Chorea erinnern, beobachtet werden. Also eine Sprachstörung, eine leichte Akinese sowie eine hyperkinetische Bewegungsstörung. Daneben ein symptomatischer Korsakoff. Wir werden nach Analogie des ersten Falles Deutsch auch bei den beiden anderen eine striäre Funktionsschädigung annehmen dürfen, die im zweiten Fall makroskopisch unbemerkt blieb, während im letzten der Grad der Schädigung so gering war, daß eine Erholung der Kernmassen möglich wurde. 
In allen Fällen kommt es zu einer psychomotorischen Störung, die Sprach- und Körpermuskulatur umfaßt, in den schweren Fällen bildet sich ein stuporähnlicher Zustand aus. Inwieweit das Syndrom des Korsakoff striär bedingt sein könnte, bleibe einer späteren Untersuchung vorbehalten. Daß dies nicht von vornherein auszuschließen ist, darauf weisen die Kohlenoxydvergiftungen hin, bei denen sowohl die symmetrische Erweichung des Linsenkerns wie das Syndrom des Korsakoff zu finden ist*). Von den übrigen Typen striärer Erkrankungen erwähne ich noch den Torsionsspasmus und die Athétose double, während wir die Chorea bei der Vielgestaltigkeit ihrer lokalisatorischen Bedingtheit außer acht lassen wollen. [Auch sind Klei sts ${ }^{23}$ ) Untersuchungen über die Chorea-Psychosen so erschöpfend, daß hier nur auf seine Arbeit verwiesen zu werden braucht.] Einen Fall von Torsionsspasmus, den wir hier beobachten, können wir in Parallele mit dem bekannten Thomallaschen ${ }^{38}$ ) Fall setzen; es handelt sich um einen im 4. Lebensjahr erkrankten jungen Menschen, mit ausgesprochenen torquierenden Bewegungsstörungen des Rumpfes und athetotischen der Extremitäten, neben denen dysarthrische Sprachveränderungen, choreatische Gesichtszuckungen, sowie grimassierende Mimik bestehen. Er zeigt psychisch Neigung zu Erregungszuständen, die früher häufiger auftraten als jetzt, neben dieser gesteigerten affektiven Reizbarkeit findet sich ein sehr auffallendes Zwangslachen, wobei übrigens ebenfalls eine Empfindung für die Zwangsmäßigkeit nicht vorhanden ist. Dabei kann nicht von einer erheblichen Intelligenzschwäche gesprochen werden, obwohl der Kranke im Assoziationsversuch die für Imbezille nach Wehrlin ${ }^{40}$ ) typische Reaktionsweise aufwies, nämlich die definitorische Reaktion; doch besteht gute Merkfähigkeit, der Kranke liest (meist religiöse Lektüre), neigt überhaupt zu religiösen Sekten, findet sich mit seiner Krankheit auf Grund religiöser Erwägungen ab u. dgl., in seinen Träumen, die er sehr plastisch schildert, spielen Visionen des Weltunterganges eine Rolle, und man hat das Gefühl, daß die Gefahr einer religiös gefärbten Wahnbildung nicht gering ist. Bemerkenswert ist die außerordentliche Geschicklichkeit, mit der der Früherkrankte feinere Bewegungen, wie Schuhe zubinden u. dgl. ausführt, und die dauernden hyperkinetischen Reize, die ja nicht nur den Rumpf, sondern auch die Extremitäten betreffen und hier zu athetotischen Bewegungen der Finger führen, überwindet. Man kann hier keineswegs von einer Verarmung

*) In einem hier zur Sektion gekommenen Fall von Alkoholpsychose mit Korsakoffschen Symptomen fand sich makroskopisch eine symmetrische Erweichung im Linsenkerngebiet, doch mußten aus technischen Gründen Untersuchung der Rinde und Serienschnitt unterbleiben, so daß Schlußfolgerungen aus dem Fall nicht gezogen werden können. 
der Willkürbewegungen sprechen, vielmehr wird der Versuch gemacht und zum Teil mit bewundernswertem Geschick durchgeführt, die intendierte Bewegung trotz aller Störungen auszuführen. Trotzdem zeigt sich die obenerwähnte Alteration der Affektivität, die in gewissem Widerspruch zu der bei der Unterdrückung der rein körperlichen Bewegungseffekte aufgewandten Energie steht. Aber es bedeutet, wie $\mathrm{Ach}^{1}$ ) ausführt, keine geringe Anforderung an den Willensapparat, affektbetonte Reize unbeantwortet zu lassen, und dieser Anforderung vermag der Kranke nicht zu genügen. Im übrigen werden die seelischen Ausfälle bei der Dysbasia lordotica nicht als regelmäßiger Befund erachtet, immerhin geht aus der Zusammenstellung von Mendel ${ }^{21}$ ) hervor, daß Depressionen, affektive Reizbarkeit, Indolenz und Apathie gefunden werden, Thomallas Kranker wurde scheu und ängstlich, später litt er an Erregungszuständen, bei Schwalbes Fall bestehen Halluzinationen und Delirien.

Kann man bei dieser Gruppe nur mit hoher Wahrscheinlichkeit den Linsenkern als Sitz der Erkrankung annehmen, so dürfte dies nach $O p$ penheims und $O$. und C. Vog $t_{s^{39}}$ ) Untersuchungen feststehen für die Athétose double, die nach diesen Autoren auf dem État dysmyelinisatus der Linsenkerne beruht. Eine Analyse der stets bei der Krankheit beobachteten Geistesstörung stößt auf große Schwierigkeiten, weil die Krankheit in frühester Jugend zu entstehen pflegt und bei den schweren Behinderungen, die das Gesamtmotorium einschließlich der Sprache erfährt, die unentwickelten Geschöpfe selten die Kraft zur intellektuellen Ausbildung aufbringen können. Auch hier beobachtet man wieder als vorwiegendes Moment neben der intellektuellen Verkümmerung die Labilität und Reizbarkeit der affektiven Zone, wir fanden bei einem Fall des Spitals direkte Wutausbrüche, auch Verwirrtheitszustände des in frühester Jugend erkrankten Geschöpfes, bei dem das Leiden zu einer grotesken Verunstaltung des ganzen Körpers geführt hat. Die Unglückliche kann durch die Verbiegungen der Wirbelsäule und die Contracturen in den großen Gelenken (Spasmus mobilis), zu denen das Leiden geführt hat, nur auf dem Bauche liegen, und es ist überraschend, daß trotz der Verunstaltung des Körpers, trotz der hochgradigen Sprachstörung von explosiv bulbärem Charakter, die eine Verständigung kaum ermöglicht, es doch zu intellektuellen Leistungen kommt, die allerdings minimal sind. Auffällt die Gefühlsbetonung, mit der die Kranke ihrer Umgebung gegenübersteht, wie wählerisch sie etwa dem Pflegepersonal gegenüber ist $\mathbf{u}$. dgl.

Nach dieser kurzen Übersicht über die seelischen Störungen bei Erkrankungen der Basalganglien, sei der Versuch gemacht, Erscheinungen körperlicher Art bei Geisteskrankheiten in Beziehung zu diesen Hirnteilen zu bringen. In Betracht kommt hier vor allem die katatone 
Form der Dementia praecox. Und es ist nicht nur die in die Augen fallende Analogie der Bewegungsstörung, es sind eine ganze Reihe körperlicher Symptome, die in gleicher Weise bei den obengenannten Nervenkrankheiten wie bei der Katatonie zu finden sind.

Um mit den niederen reflektorischen Erscheinungen zu beginnen, seien zunächst die Anomalien der Pupillarreflexe genannt, auf die A. Westphal kürzlich von neuem hinwies. Es werden bekanntlich Ungleichheiten der Pupillen, Trägheit der Reaktionen, auffallende Weite, Verziehungen und vor allem Ausbleiben der Beantwortung psychischer Reize beobachtet. Löwenstein ${ }^{24}$ ) sieht als Ursache an „,den Verlust der Spontaneität bei erhaltener Suggestibilität des Gefühlslebens", eine psychologische Erklärung, die an sich richtig sein möge, für das körperliche Phänomen mir nicht zwingend erscheint. Nimmt man jedoch eine Erkrankung der zentralen Ganglien an, so ist die Beteiligung der Pupillenreflexbahn anatomisch ungezwungen erklärt, denn der Hypothalamus, das Zentrum des Sympathicus, steht durch die Ansa lenticularis mit dem Linsenkern in enger Verbindung*). In gleicher Weise lassen sich auch die schweren vasomotorischen, mitunter bis zu Paroxysmen gesteigerten Alterationen verstehen, Schweißausbrüche profuser Art, Cyanose der Extremitäten, Pulsschwankungen, ja Ödeme kommen häufig vor, sind als Reizerscheinungen der vegetativen Zentren jedenfalls unserem Verständnis nähergebracht, als durch psychologische Ausdeutung. Schließlich sei auch der splanchnischen Erscheinungen gedacht, die sich in Darmstörungen, launenhaftem Wechsel der Urinmengen usw. bemerkbar machen. Auch der zuweilen geradezu profuse Speichelfluß gehört in die Reihe der vegetativen Funktionsstörungen. All diese Symptome finden wir wieder bei den subcorticalen Nervenkrankheiten. Verdauungsstörungen, Schweißausbrüche, Speichelfluß gehören durchaus in das Bild des Morbus Wilson und der ihm verwandten Zustände**).

Von besonderem Interesse muß es sein, die innersekretorischen Drüsenstörungen unter dem Gesichtspunkt ihrer zentralen Innervation

*) Die Reaktion der Pupillen auf indirekte Reize kann als feinster Indicator der ungestörten Reflextätigkeit angesehen werden. Ihr Ausfall, der ja am häufigsten zur Beobachtung kommt, könnte als Zeichen einer für unsere Mittel anatomisch nicht faßbaren, dynamischen Beeinträchtigung angesehen werden. Übrigens werden Pupillarstörungen, z. B. Anisokorie, von Mingazzini ${ }^{30}$ ) zu den Kardinalsymptomen der Linsenkernerkrankung gerechnet.

**) Eine gute Übersicht und klare Auseinandersetzung über die Reihe der vegetativen Störungen bringt Bö $\mathrm{nheim}^{5}$ ), er weist die Wahrscheinlichkeit einer cerebralen Beeinflussung der Gallensekretion an der Störung der Gallenfarbstoffausscheidung nach. Er stellt das Erfahrungsmaterial der verschiedenen Beobachter bezügl. der Darmstörungen, der Magensekretionsanomalien zusammen und erläutert ausführlich die Momente, die für eine Abhängigkeit der Miktion und De- 
zu verfolgen. Seit Fa user ${ }^{11}$ ) die Abderhaldensche Reaktion bei Geisteskranken anwandte, kann das Vorhandensein einer innersekretorischen Dysfunktion bei der Dementia praecox als gesichert gelten; auch bei den striären Erkrankungen ist sie häufig zu finden, und $O p$ penheim ${ }^{29}$ ) erhoffte von der Anwendung des Dialysierverfahrens wichtige Aufschlüsse für ihre Genese. Hier wie dort äußern sie sich in einer Beeinträchtigung der Geschlechtsdrüsen, Sistieren der Menses, Nachlassen der Potenz, in Veränderung der Haut*), Pigmentanomalien, Stoffwechselstörungen (Fettansatz bzw. Abmagerung). Es ist nun durchaus wahrscheinlich, daß der innersekretorische Apparat genau so einer nervösen Beeinflussung unterliegt, wie alle übrigen Körperorgane. Wie wäre sonst das Auftreten vermehrter Adrenalinproduktion durch Schockwirkung, wie die seelische Beeinflußbarkeit der Thyreoidea, der Ovarialfunktion zu erklären? Daß die innervatorische Regulation durch das Striatum erfolgt, für diese Auffassung ist Frank mit guten Gründen eingetreten. [Die Dysfunktion der Blutdrüsen wird sich sekundär durch den Metaprozeß (Fauser) zu einer Gefahr für den Körper entwickeln. Fauser versteht darunter die Schädigung durch die Abwehrfermente, die von dem Körper gegen die Abbauprodukte der krank eingestellten Organe produziert werden. Die Abwehrfermente werden ihrerseits mithelfen, das schon aus dem Gleichgewicht gebrachte Zentralorgan zu schädigen. Ich möchte hypothetisch darauf hindeuten, ob nicht die bei Katatonikern gefundenen und auch von Goldstein ${ }^{18}$ ) als sekundär aufgefaßten Rindenveränderungen durch solche Metaprozesse bedingt sind.]

Welches der beiden vegetativen Systeme primär und hauptsächlich erkrankt ist, kann bei dem ungeklärten Stand des Tonusproblems von Vagus und Sympathicus nicht gesagt werden, anzunehmen ist eine Affiziertheit beider, sei es, daß diese zustandekommt durch direkte Beeinträchtigung beider Zentren, etwa des Linsenkernes als das für das autonome, des Hypothalamus als das für das sympathische System, sei es dab indirekt die Ausschaltung des einen zur gesteigerten und so unzweckmäßigen Funktion des anderen führt und so eine Summation der Wirkungen sich bemerkbar macht. (Hier bedarf es noch genauer Untersuchungen.)

Daß auch psychische Reize die hier erwähnten körperlichen Erscheinungen hervorrufen können, daß sie ,psychogen" bedingt sein

fäkation von den subcorticalen Ganglien sprechen. Hier sei erwähnt, daß von einem italienischen Autor als häufigster pathologisch-anatomischer Befund an den inneren Organen von Schizophrenen Leberveränderungen vorliegen. Jedenfalls wären genaue Funktionsprüfungen der Leber bei Katatonen von Wichtigkeit.

*) In dem von mir beschriebenen Fall von traumatischer Pseudosklerose entwickelte sich eine typische Fischhaut. Bei Katatonischen sind sehr häufig Chloasmen u. a. Hautveränderungen zu finden. 
können, braucht nicht als Einwand angesehen zu werden, denn schließlich bedarf der seelische Reiz auch eines Angriffspunktes, um zur körperlichen Manifestation zu führen.

Daß dieser Angriffspunkt in den tiefergelegenen Hirnteilen, wah ${ }^{-}-$ scheinlich in den Basalganglien zu suchen ist, zu dieser Auffassung führt vor allem die Analyse der auffälligsten der somatischen Erscheinungen, der Bewegungsstörungen. Wies jüngst in seiner geistvollen Studie über die Willensapparate der Hysterischen Kretschmer ${ }^{25}$ ) auf die inneren Beziehungen der Haltungsstörung des Katatonen zu der des Wilson-Kranken hin, so sind es von früheren Untersuchungen über die Bewegungsstörungen bei Geisteskranken vor allem die Arbeiten von Kleist ${ }^{22}$ ), in denen ein Versuch zu lokalisatorischen Bestimmungen gemacht wurde. Bekanntlich hat Kleist angenommen, daß die Stirnhirn-Kleinhirnbahn, und zwar sowohl ihre aufsteigende wie die absteigende Leitung bei den psychomotorischen Bewegungsstörungen geschädigt ist. Vor allem sieht Kleist in dem Frontalhirn, und zwar seinem Rindenteil, dem Ursprungsgebiet der cerebellocorticalen Bahnen, den Sitz der Erkrankung. Hinweisend auf diese Lokalisation sind ihm nicht nur die Ergebnisse der Analyse der körperlichen Erscheinungen, sondern auch die Betrachtung der seelischen Ausfälle. Für die ersten wird die Verbindung des hebephrenen Prozesses mit dem choreatischen Symptomenkomplexes bzw. mit Нypotonie und Ataxie vom Charakter der Stirnhirnataxie angeführt. Die Bewegungsstörung besteht hauptsächlich in einer innervatorischen, in der nach Kleist die Unfähigkeit zur kinetischen Innervation bei Erhaltung der statischen Innervationsfähigkeit das Charakteristische ist. Die Spannungserscheinungen werden als sekundär angesehen, in Analogie der Steigerung der Sehnenreflexe nach Pyramidenbahnlähmung. Erwähnt werden wiederholt die bei Thalamuserkrankungen beobachteten Erscheinungen und eine funktionelle Einbeziehung des Sehhügels durch den Rindenprozeß als möglich hingestellt. Der Widerspruch in den gleichzeitig vorhandenen Erscheinungen eines gesteigerten Tonus wie einer Hypotonie soll dadurch erklärt sein, daß die ataktisch-hypotonisch-choreatischen Symptome der aufsteigenden Kleinhirn-Stirnhirnbahn, die tonischen der absteigenden Stirnhirn-Brücken-Kleinhirnbahn angehören. Es handelt sich um eine transcorticale Ausschaltung des Systems aus seinen Verbindungen.

Auch die begleitenden Störungen sympathischer Reflexe (Herz, Puls, Atmung) werden auf die Alteration der Stirnhirnrinde zurückgeführt. Die psychologische Analyse der psychomotorischen Störungen führen Kleist zu der Annahme ihrer Abhängigkeit von Organempfindungen bzw. höheren seelischen Bildungen (Gefühle, Affekte, Aufmerksamkeit), die ,ebenfalls in bestimmten Bestandteilen dem Komplex 
der Organempfindungen, dem Bewußtsein der Körperlichkeit angehören“. Die Denkstörung bei psychomotorisch Geisteskranken ist abhängig von dem Grad der psychomotorischen Störung selbst. Der Ausfall der Einstellungsbewegungen führt zu Schädigungen des Wahrnehmungsaktes, zur Merkschwäche, zur Verlangsamung des Denkaktes, zu assoziativen Fehlleistungen. Daneben vertritt dann noch verstärkend der Ausfall von Gefühlen. Der Negativismus verbindet sich oft innig mit den Spannungserscheinungen.

Wie die akinetischen Zustände werden auch die hyperkinetischen auf eine zentral gelegene Schädigung der obengenannten Leitungssysteme zurückgeführt; bei den Denkstörungen kommt es zu einem circulus vitiosus. Erst bedingt die Bewegungsstörung kraft der Schädigung der Einstellvorgänge eine Denkstörung und diese steigert ihrerseits wieder die Neigung der Kranken, sich bald nach dieser, bald nach jener Richtung hin flüchtig einzustellen und in kurzläufigen Reaktionen auf die zufälligen äußeren Eindrücke zu antworten. Dies in kurzen Zügen über die Kleistsche Theorie der Bewegungsstörungen. Natürlich enthalten die Arbeiten eine Fülle von hervorragend beobachtetem Einzelmaterial, auf das hier nicht eingegangen werden soll, ebensowenig wie auf die Abgrenzung gegen die organischen Sprach- und Bewegungsstörungen bei Aphasischen und Apraktischen, um die er sich bemüht.

Wie nahe die vorliegende Arbeit der Tendenz von Kleists Untersuchungen liegt, erscheint wohl klar. Deshalb seien einige prinzipielle Einwendungen erlaubt, die sich nicht gegen die mir vorbildliche Richtung der Untersuchungen, sondern gegen ihre Folgerungen wenden. Schon eine der Grundbeobachtungen, die Kleist erwähnt, erscheint mir zweifelhaft: Ist die Bewegungsstörung des Schizophrenen wirklich vorwiegend eine kinetische? Gerade hier wird uns, so meine ich, die Analogie mit den subcorticalen Ganglienerkrankungen weiterbringen. Auch da sehen wir eine Akinese, einen Bewegungsausfall, der oft, aber doch nicht immer mit einem Hypertonus verknüpft ist.

Schon den Neurologen fiel die Bewegungsarmut in den Fällen von striärer Erkrankung auf und sie betonten, daß eine Parallelität von Ausfall an Bewegungen mit Spannungserscheinungen durchaus nicht immer festzustellen ist. So schreibt Cassire ${ }^{6}$ ), daß ,,ein anderes Moment als die erschwerte Ansprechbarkeit der Agonisten und Antagonisten zur Erklärung der Bewegungsarmut heranzuziehen sei". (Rieger erklärte die Katalepsie durch gleichstarke Innervation von Protagonist und Antagonist, zit. n. Ble uler.) Wir haben angeführt, daß wir dieses Moment sehen in einer Schwächung des Antriebes, der Spontaneität, der psychischen Aktivität. Wenn Kleist jedoch als charakteristisch für die Bewegungsstörung des Geisteskranken die Akinese bei erhaltener 
statischer Innervation sieht und daneben gerade die Symptome hervorhebt, wie choreatische Zuckungen, Tremor, häufiger Hypertonus, die Strümpell unter dem amyostatischen Symptomenkomplex vereinigt hat, so sind diese Tatsachen schwer in Einklang zu bringen. Geht man aber mit Berze davon aus, daß in den subcorticalen Ganglien der Impuls zur motorischen Umsetzung erfolgt, die Innervation der intentionalen Felder, so ist der Widerspruch gelöst, denn gleichzeitig sind diese Hirnteile, wie es die klinische Beobachtung unstreitbar beweist, auch für das Zustandekommen des motorischen Aktes selbst von Bedeutung, sie regeln die Synergien. Natürlich verlangt ein starker Hypertonus zu seiner Überwindung einen größeren Kraftaufwand als der Bewegungsvorgang des Normalen, aber wir sehen ja, daß bei Intaktheit des Zentrums für den Antrieb dieser Widerstand überwunden wird in allen Fällen von Pyramidenbahnerkrankungen. Man kann also nicht von einer Intaktheit der statischen Innervation, genau so wenig wie der kinetischen Innervationen bei Katatonikern sprechen, vielmehr besteht primär eine Schwächung der Innervationstendenz überhaupt und daneben findet sich sekundär häufig eine, fast könnte man sagen mechanische oder besser physiopathologische Störung der Myostatik, viel häufiger wenigstens als der Kinetik. Denn gelingt es durch körperliche oder seelische Reize, die primäre Willensschwäche des Katatonen zu überwinden, so ist der kinetische Vorgang meist ein ungestörter, während die statische Beeinträchtigung (choreatische Zuckung, Zittern, Spannung der Muskulatur) von dem Willen an sich ganz unabhängig zu sein scheint.

Hierzu zwei Beobachtungen. Durch Überrumplung mit faradischem Strom konnte ich katatone Kranke, die wochenlang mutacistisch waren, vorübergehend zum Sprechen bringen (wie übrigens auch der ursprüng. lich als hysterisch verkannte Patient Economos, ein Fall von WilsonMorbus durch solche Behandlung seine Sprachhemmung ,"überwandt", natürlich auch nur passager).

Uberhaupt zeigt die innervatorische Komponente der Sprache alle Erscheinungen der übrigen motorischen Störungen; sie sind ihnen genetisch gleich zu beurteilen. Das dysarthrische, das abgehackte Sprechen steht auf gleicher Linie wie etwa die in einzelnen zagen ruckweisen Etappen erfolgende Bewegung vieler Kranken, wenn sie aufgefordert werden, die Hand zu geben, oder wie die Asynergie beim Gehen, wie Bleuler sie in seiner Monographie beschreibt. (Man könnte auch hier mit Kleist von einem Zerfall des Bewegungs- bzw. Sprechvorganges in seine Bausteine sprechen. Und bestätigt sich die neuerdings stark hervorgehobene Ansicht von der doppelten Innervation der Muskulatur, der contractilen Fibrillensubstanz durch die motorischen, der „Haltesubstanz", des Sarkoplasmas, durch sympathische bzw. parasympathische 
Nerven [Frank $\left.\mathbf{k}^{15}\right)$ ], so wäre hier auch ein Weg, den Zerfall der Synergien anatomisch genauer zu fassen, die hyperkinetischen Erscheinungen etwa durch eine Hypofunktion, die Spannungen durch einen Reizzustand des Innervationszentrums der parasympathisch regierten ,Tonusmuskeln“ zu erklären.)

Ist die Kinetik nun durch äußere Beeinflussung - wenn auch nur vorübergehend - in der Richtung zur Norm regulierbar, so versagt bei den statischen Innervationsstörungen jedes Mittel. Eine junge Hebephrene z. B., mit der eine Verständigung noch möglich war, zeigte im Verlauf der Krankheit anfallsweises Auftreten von Schnauzkrämpfen mit choreiformen Gesichtszuckungen, besonders in der Unterhaltung. Bleuler hat bekanntlich den Schnauzkrampf psychologisch als Zeichen der Verachtung zu deuten gesucht. Dem widerspricht schon das anfallsweise Auftreten. Erklärbar erscheint er mir eher als dystonisches Symptom. Die zum Sprechakt tendierte Innervation entgleist, die Stellung wird aber - eine Parallelerscheinung der Fixationsrigidität an den Extremitäten - festgehalten.

Diese Erklärung enthebt uns auch der unbefriedigenden Lösung, die hypertonischen Erscheinungen auf die absteigenden, die hypotonischen auf die aufsteigenden Bahnen zu verteilen und überhaupt den Grad der vorhandenen Bewegungsstörungen von der mehr oder weniger weitgehenden Beteiligung der Bahnen des Kleinhirnsystems abhängig zu machen. Allerdings fällt auch die Kleistsche Folgerung, daß die Denkstörungen sekundär als Folge der Bewegungsstörungen aufzufassen sind. Es ist nicht einzusehen, warum der Ausfall der Empfindungen von Einstellbewegungen auf den Denkakt von so entscheidendem Einfluß sein soll. Es kommen doch Paraplegien zur Beobachtung, die keineswegs eine Erschwerung der Denkakte erkennen lassen. Bei allen Systemerkrankungen, bei der Tabes mit ihrem Ausfall der Tiefensensibilität, müßten dann doch schwere Beeinträchtigungen der intellektuellen Leistungen beobachtet werden. Das ist aber durchaus nicht der Fall. Wie oft sind etwa Lähmungen der Augenmuskeln, die ja für die Einstellung, für die Aufmerksamkeit von großer Bedeutung sein müßten, zu beobachten, ohne daß der Wahrnehmungsakt als solcher hierdurch beeinträchtigt wird. Auch bei der Erklärung der katatonen Denkstörungen scheint uns der intentionale Faktor vorwiegend als geschädigt in Betracht zu kommen und muß vor allem herangezogen werden. Antrieb gehört zum Denkakt, genau wie zu jeder motorischen Umsetzung, und nicht die Einstellungsbewegung führt zur Aufmerksamkeit, sondern die Auf. merksamkeit die aktive Apperzeption zur Einstellung.

Damit ist die Bedeutung der Organempfindungen gewiß nicht bestritten; sie ist ja besonders für die Verstärkung von Affekten, 
überhaupt für die Intensitäts-Projektion unserer Gefühle und Vorstellungen erheblich. Nur ihr Primat wird nicht anerkannt.

Wir kommen also zu dem Schluß, daß als Angriffspunkt der unbekannten Noxe, die zur Katatonie führt, die subcorticalen Ganglien in Betracht zu ziehen sind, vor allem das Striatum, dessen Betroffensein aber, wie schon Wilson anführt, auch für den Thalamus opticus von wichtigster dynamischer Wirkung sein könnte. Indem wir diese Hirnteile als den primären Sitz dèr cerebralen Erkrankung ansehen, erkärt sich uns der katatone Symptomenkomplex in seinen seelischen und körperlichen Manifestationen, die seelischen weisen das Grundsymptom der intentionalen Schwäche auf, was sich verstehen läßt, wenn man nicht die Hirnrinde als Sitz der seelischen Grundfunktionen ansieht, sondern in die grauen Massen des Subcortex den Punkt verlegt, von dem aus die "Tonisierung" unserer Psychismen ausgeht; die körperlichen, wenn man in jenen Hirnteilen Zentren oder Schaltstationen all jener körperlichen Mechanismen ansieht, die oben aufgeführt sind und deren Störung ja in der Tat zu völlig analogen Erscheinungen führt, die uns die Neurologie der striären Erkrankungen lehrt.

Hierbei sei betont, daß auch nur andeutungsweise zu erklären, warum sich in katatonischen Erkrankungen die Symptomatik so verschieden ausprägt, uns heute ebenso unmöglich ist, wie der gegenwärtige Stand der neurologischen Forschung eine Deutung gestattet, warum wichtige Symptome wie Dysarthrie oder Blasenstörungen oder Contracturen in dem einen Fall von Pseudosklerose vorhanden sind, in dem anderen fehlen. Die Topographie dieser Hirnteile ist eben trotz der grundlegenden Arbeiten Marburgs, Mingazzinis, C. und o. Vogts u. a. erst im Werden, und ebenso die Pathophysiologie. Auf die Diskussion mancher strittiger Einzelheiten bin ich daher nicht eingegangen, auch die Beziehungen zu den Erkrankungen des Kleinhirns und anderen heredo-degenerativen Prozessen (atrophie olivoponto-cérébelleuse) wurden außer acht gelassen, obwohl auch sie, wie Stauffenbergs Fall zeigt, psychiatrisch bedeutungsvoll sind (diese Zeitschr. Bd. 39). Auch auf die Erklärungsversuche der Einzelarten der Bewegungsstörungen wie Chorea, Tremor, Athetose konnte kein Bezug genommen werden. Abgesehen von ihrer Eigenfunktion liegt die besondere Bedeutung der Stammganglien auch hier in ihrer anatomischen oder dynamischen Verknüpfung mit der Gesamtheit der übrigen Hirnteile. In psychiatrischer Hinsicht werden die Auffassungen über die psychopathologischen Erscheinungen der Wahnbildung und der Trugwahrnehmungen durch unsere Erörterungen nicht berührt, sie treten aber an Bedeutung in den hier betrachteten Krankheitsbildern zurück. (Die Scheidung der katatonen von der paranoiden Form der Schizophrenie, die auch Bumke fordert, sollte bei allen Unter- 
suchungen sowohl klinischer wie anatomischer Art durchgeführt werden.) Ohne eine Hierarchie der psychischen Gebilde aufzustellen oder sie gar mit anatomischen Punkten in Parallele setzen zu wollen derart, daß ein „Höher" im Seelischen zugleich in den oberen Stockwerken des Gehirnes zu suchen ist, kann doch gesagt werden, daß die Loslösung des Cortex von dem Stamm (die übrigens schon Anton zur Erklärung der Katalepsie heranzog, zit. nach Bleuler), sich in psychologischer Hinsicht durch den Triumph primitiver Funktionen über komplizierte geltend machen wird. Aber gerade weil die verwickelten seelischen Vorgänge sich auf den einfachen aufbauen, werden ihre Störungen manifest. Die innige Verknüpfung der Gefühle z. B., auch der erhabenen, mit dem Somatischen wird zu ihrer Alteration führen, wenn zugleich mit psychischen Grundfunktionen die vegetation Zentren betroffen sind.

Ein anderes Beispiel: Die rhythmische Bewegung (also auch die sprachliche) verlangt nach Jelgers $\mathbf{m a}^{20}$ ) nur einen niederen Grad der Koordination, ist also als die primitive anzusehen. Nun zeigen, wie Fauser ${ }^{12}$ ) hervorgehoben hat, gerade Katatoniker oft rhythmische Betonung.

Die psychologische Erklärung, die Fauser dafür gibt, ist die, daß die rhythmische Betonung als pathologisches Symptom nichts anderes als ein Zurücktreten der aktiven Aufmerksamkeit und Willensvorgänge hinter den sinnlichen Antrieben bedeutet. Also auch hier ein Hervortreten primitiver Funktionen, das anatomisch durch den Ausfall hem. mender oder erregender Einflüsse bestimmter Hirnteile bedingt ist, psychologisch sich auf die Insuffizienz der seelischen Grundfunktion zurückführen läßt.

Es sei gestattet, aus dem zur Verfügung stehenden klinischen Beobachtungsmaterial, insbesondere der symptomatischen Psychosen noch einiges anzufüh'en, was die Wahrscheinlichkeit der Darlegungen zu stützen geeignet ist. Hier gilt es vor allem, der Encephalitis lethargica zu gedenken, deren epidemisches Auftreten in den letzten Jahren wesentliche Feststellungen erlaubte. Bekanntlich hat die pathologischanatomische Untersuchung der Lethargica das vorwiegende Betroffensein der Basalganglien nachgewiesen. Dem entsprachen auch die Befunde zahlreicher Beobachtungen, sowohl in körperlicher wie in seelischer Hinsicht. Schon die Schlafsucht ist ja ein Phänomen, das man körperlich wie seelisch auffassen kann. Über die Theorie des Schlafes sind an dieser Stelle gewiß keine Erörterungen angängig, erwähnt sei nur, daß Trö m m ner im Thalamus den Ort der Schlafentstehung sieht. (So wäre auch die häufige und mitunter sehr intensive Schlafsucht der Katatonen zu verstehen.) Auch im übrigen war die Symptomatik der Encephalitis dem katatonen Zustandsbild in einer Weise analog, daß 
Verwechslungen in der Diagnosenstellung gar nicht selten waren, daß zahlreiche Encephalitisfälle unter der Diagnose der Dementia praecox dem Spital eingeliefert wurden. So spricht Strüm pell geradezu von einer katatonen Form der ,Hirngrippe“. Auch in der weiteren Beobachtung der chronischen Formen der Encephalitis zeigte sich eine große Übereinstimmung der beiden in Betracht kommenden Krankheitsbilder. Die Bewegungsarmut in diesen Fällen bleibt eine dauernde, so daß die Bezeichnung einer "Encephalitis amyostatica" (W. Cohn) mit Recht gewählt werden kann. Gleichzeitig ist auch die gemütliche Reizbarkeit, sowie der Mangel an Antrieb in diesen sich lange Zeit hindurchziehenden Zustandsbildern ein bleibender Defekt. Neben der affektiven Reizbarkeit besteht aber auch, wie wir dies wiederholt beobachten konnten; eine gemütliche Stumpfheit, die sich von der des Katatonikers gewiß nicht unterscheiden läßt. Was die intellektuelle Leistungsfähigkeit dieser Kranken betrifft, so kann man von einer Verblödung nicht direkt sprechen, sondern ebenso wie in den oben geschilderten Fällen von Wilsonscher Krankheit ist die Ansprechbarkeit "die Aktivierung" der zur intellektuellen Leistung notwendigen Zonen äußerst erschwert und es bedarf besonderer Stimulantia, sie in Tätigkeit zu setzen. Wenn nun auch der infektiöse Prozeß die Rinde nicht verschont, [ich verweise statt Literaturangaben auf die Originalarbeit von Economo $o^{19}$ )], so sind es doch vor allem die grauen Kernmassen, die Veränderungen aufzeigen. Aber nochmals sei betont, daß die Deutung von Befunden pathologisch-anatomischer Art in der Rinde, gerade was ihre psychologische Verwertbarkeit betrifft, nicht dazu führen darf, in dem Betroffensein des Cortex den Grund der psychischen Störungen zu sehen. Zum mindesten darf B erzes Einwand (l. c.), nicht übersehen werden, daß die Rinde nicht nur die Aufgabe der Umsetzung der sensorischmotorischen Impulse hat (Impressionsfelder), sondern in ihrer Gesamtheit Träger der intentionalen Funktion ist, die im Gegensatz zu der ersteren lokalisatorisch nicht in verschiedene Felder mit spezifischen Leistungen getrennt werden kann. Diese intentionale Sphäre setzen wir in Abhängigkeit zu den Basalganglien, und selbst bei scheinbar nur durch Veränderungen der Hirnrinde in pathologischer Hinsicht gekennzeichneten Krankheitsbildern muß sich künftig eine scharfe Trennung herauskrystallisieren lassen, welche der beiden Sphären als geschädigt anzusehen sind, die in Einzelfelder auflösbare impressionale oder die über die Gesamtoberfläche sich erstreckende intentionale. In ihrer Gesamtheit dürften die Ergebnisse aus der Encephalitisepidemie eine starke Stütze sein für die Auffassung der sog. exogenen Reaktionsformen bei symptomatischen Psychosen, insbesondere für Bonhoeffers Ansicht über die Unspezifität der katatonischen Symptome. Fast scheint es, daß man dahin kommen muß, die Katatonie als isoliertes Krank- 
heitsbild überhaupt aufzugeben und nur von einem kata. tonen Symptomenkomplex zu sprechen, der unter den ver. schiedensten Bedingungen entstehen kann. Daß endogene Faktoren auch hierbei nicht auszuschließen sind, bedarf wohl keiner Erwägung. Angeführt sei ein Fall von ausgesprochener Katatonie, der entstand, als gleichzeitig der Bruder der Kranken mit typisch encephalitischen Symptomen in ein anderes Spital aufgenommen war. Hier spricht schon die schwere erbliche Belastung der Kranken (zwei Brüder des Vaters und einer der Mutter sind geisteskrank) für eine konstitutionelle Schwäche des Gehirns, welches auf Noxen verschiedentlicher Art allerdings nur darum in gleicher Weise antworten wird, wenn diese Noxen selbst in ihrer Affinität zu bestimmten Hirnteilen gleichgerichtet sind. In der Tat ist dies auch der Fall. Wie die Erreger der Encephalitis lethargica bzw. ihrer Toxine vorzüglich die grauen Hirnmassen ergreifen, so sehen wir, daß Gifte, wie das Leuchtgas, das Mangan, wahrscheinlich auch das Jodoform zu diesen Hirnteilen besondere Wahlverwandtschaft zeigen und es daher bei derartigen Intoxikationen zu den Bildern der amyostatischen bzw. katatonen Erkrankung kommt. Eine Erklärung dieser Tatsachen ist uns heute nicht möglich. Wie mir scheint, liegt sie auf dem Weg der physikalisch-chemischen Forschungsrichtung, und vielleicht kann das Ergebnis des Chemikers [A. Weil'11)], daß die graue Hirnsubstanz eine größere Quellungsfähigkeit besitzt als die weiße, zu solcher Deutung herangezogen werden*), besonders wenn man bedenkt, daß die Eigenart der Gefäßverteilung der Basalganglien zu Schwankungen der Körpersäfte erheblich disponiert. Kolis $\mathrm{ko}^{24}$ ) hat die Eigenart der Blutversorgung gerade der Linsenkerngebiete hervorgehoben und sie als prädestiniert zu Störungen in der Blutversorgung bezeichnet, weil sie versorgt werden von außerordent. lich zarten Arterien, deren Verlauf rückläufig im spitzen Winkel erfolgt, bzw. in senkrechter Abzweigung. In diesem Zusammenhang gewinnen die obenerwähnten Befunde von einer auffallend harten Hirnkonsistenz bei Pseudosklerose eine besondere Bedeutung, und ebenso die durch Reichardts Verdienst als Hirnschwellung erkannte Zustandsänderung der Hirnsubstanz, die ja nach diesem Autor in einer Änderung des Kolloidalzustandes des Gehirns beruht und nicht das Gesamtorgan ergreifen muß, sondern sich auf Teile beschränken kann. Aber wie weit zurück sind wir in diesem Gebiet noch an eindeutigem Beobachtungsmaterial. Wie isoliert in der Literatur der Dementia praecox stehen Arbeiten wie die Pötzels ${ }^{33}$ ) über Hirnschwellung oder St. Rosenthal ${ }^{34}$ ) in Nissls Beiträgen veröffentlichte pathologisch-anatomische Untersuchung, bei der im übrigen außer der Hirnschwellung

*) Phigini ${ }^{32}$ ) findet ebenfalls bei der chemischen Analyse u. a. eine Vermehrung des Wassergehaltes bei Gehirnen von Dem.-praec.-Kranken. 
Veränderungen in den Zellen gefunden wurden, und zwar waren bei dem akut erkrankten und plötzlich verstorbenen Katatoniker die Rindenveränderungen nicht schwerer als die des Corpus striatum und des Pons. Bedenkt man, wie empfindlich und reizbar der Säftekreislauf, dieses komplizierte System der Durchströmung des Gehirns ist, wie alle Arten infektiöser oder toxischer Reize zu Meningitiden seröser Art im Sinne Quinkes führen können, so sieht man, daß wirklich die Mög. lichkeit exogener Schädigung jener empfindlichen und empfänglichen Hirnteile so vielfach gegeben ist, daß dem gegenüber der endogene Faktor, der doch schließlich u. a. auch in einer Gehirndisposition zu suchen ist, in seiner Bedeutung für die Entstehung der katatonen Zustandsbilder erheblich zurückbleibt. Diese Erkenntnis hat wohl auch Ble uler (l. c.) dahingeführt, den Gedanken nicht abzuweisen, die Schizophrenie könne eine Nachkrankheit gewisser Infektionen sein. Die Krankheit könnte aber auch direkt durch eine oder mehrere spezifische Infektionen hervorgerufen sein, die chronisch auftreten und lange Zeit latent bleiben. Einen Einwand gegen die hier formulierten Gedankengänge zur Katatoniefrage gilt es noch zu entkräften. Nämlich daß das Grundsymptom, die Aboulie, die Willensschwäche, die Antriebslosigkeit doch als Herdsymptom zu beobachten ist, besonders bei Tumoren des Stirnhirns. So beschreibt auch Forster ${ }^{14}$ ) solch einen Mangel an Antrieb (neben Agrammatismus) nach Stirnverletzung mit kataleptischen Symptomen. Eine Kritik dieser Anschauung wird besonders darauf hinzuweisen haben, daß sich sowohl bei Verletzungen, wie bei Hirntumoren die Abgrenzung der allgemeinen Schädigung des Gehirns, wie sie schon durch die bedeutungsvollen Zirkulationsänderungen hervorgerufen werden, von dem Herdausfall schwer vollziehen läßt. Im übrigen aber sind die Erscheinungen gerade der Stirnhirntumoren durchaus nicht einheitlich, und ohne daß eine Regel erkennbar ist, werden die gerade hier wichtigen Störungen der psychischen Aktivität, der spontanen Regsamkeit ebenso oft vermißt, wie sie gefunden werden. Ich verweise auf die Zusammenstellung von Ster ${ }^{36}$ ). Im übrigen sprechen die seltenen Fälle von Tumoren der Basalganglien für die enge Beziehung, die besonders der Thalamus opticus zu den motorischen Äußerungen der Affektivität hat. Ist es ja schon Nothnagel gewesen, der gefunden hat, daß bei Thalamusherden die feinen Äußerungen der Affekte mimisch verlorengehen.

\section{Zusammenfassung.}

Die psychischen Störungen bei Erkrankungen der subcorticalen Ganglien gehen in verschiedentlichster Abstufung ihrer Intensität am meisten in die Richtung katatoner Zustandsbilder. Umgekehrt weisen die kör- 
perlichen Symptomeder Katatonie daraufhin, daB die Hirnganglien der Angriffspunkt jenerzahlreichen Noxen sind, die zu ihrer Entstehung führen können. Hiermit steht jene Auffassung im Einklang, die in der Schwächung der psychischen Aktivität das Grundsymptom der Dementia praecox sieht.

\section{Literaturverzeichnis.}

1) Ach, Wille und Temperament. - 2) Bause, Zeitschr. f. Psych. r1, 357. - 3) Berze, Die primäre Insuffizienz der psych. Aktivität. 1914; Zeitschr. f. Psych. 75, H. 2. - ${ }^{4}$ ) Bleuler, Dementia praecox im Handb. d. Psych. $-{ }^{5}$ ) Bönheim, Zeitschr. f. d. ges. Neurol. u. Psychiatr., Orig. 60. $-{ }^{6}$ ) Cassirer, Neurol. Centralbl. 1913, S. 1284. - ${ }^{7}$ ) Cohn, W., u. La uber, Münch. med. Wochenschr. 1920, Nr. 24. - $\left.{ }^{8}\right)$ De u t s c h. Jahrb. f. Psych. 37, H. 2. - ${ }^{9}$ ) Econo mo, Zeitschr. f. d. ges. Neurol. u. Psychiatr., Orig. 45. - $\left.{ }^{9}\right)$ Eco no mo, Die Encephalitis lethargica 1917. - ${ }^{10}$ ) Edinger, Lehre vom Bau und Verrichtungen des Nervensystems, 1912. - ${ }^{11}$ ) Fa user, Dtsch. med. Wochenschr. 1916. F a u s e r, Zeitschr. f. Psych. 70. 1913. F a user, Arch. f. d. ges. Psychol. 59, H. 1. - 12) Fauser, Zeitschr. f. Psych. 62, 687. - ${ }^{13}$ ) Fleischer, Dtsch. Zeitschr. f. Nervenheilk. 54. - ${ }^{14}$ ) Fors ter, Monatsschr. f. Psychiatr. u. Neurol. 46, 1. - ${ }^{15}$ ) Frank, Berl. klin. Wochenschr. 1919, Nr. 31 u. 1920, Nr. 52. - $\left.{ }^{16}\right)$ Frankl - Hochwart, Obersteiners Arbeiten 10. - ${ }^{17}$ ) Frän kel, Dtsch. med. Wochenschr. 1920, Nr. 35. $\left.-{ }^{18}\right)$ Golds tein, Neurol. Centralbl. 1910, S. 544. - ${ }^{19}$ ) Höss slin - Alzheimer, Zeitschr. f. d. ges. Neurol. u. Psychiatr. 8. - ${ }^{20}$ ) Jelgers ma, Journ. f. Psychol. u. Neurol. 23. - ${ }^{21}$ ) Kastan, Arch. f. d. ges. Psychol. 60. - ${ }^{22}$ ) Kleist, Untersuchungen zur Kenntnis der psychomotorischen Bewegungsstörungen der Geisteskranken. Leipzig 1908, weitere Untersuchungen Leipzig 1909. - ${ }^{23}$ ) Kleist, Zeitschr. f. Psych. 64, 769. - 24) Kolisko, Wien. klin. Wochenschr. 1893, Nr. 11. - ${ }^{25}$ ) Kretsch mer, Zeitschr. f. d. ges. Neurol. u. Psychiatr., Orig. 54. $-{ }^{26}$ ) Löwenstein,Monatsschr. f. Psychiatr. u. Neurol. 4\%, 194. — ${ }^{27}$ ) Maas, Neurol. Centralbl. 1918. - ${ }^{28}$ ) Mende1, Monatsschr. f. Psychiatr. u. Neurol. 46. ${ }^{29}$ ) Me yer, E., Med. Klinik 1910 - ${ }^{30}$ ) Mingazzini, Zeitschr. f. d. ges. Neurol. u. Psychiatr., Orig. 8, H. 1. - ${ }^{31}$ ) O p penheim, Dtsch. Zeitschr. f. Nervenheilk. 56, 332. - ${ }^{32}$ ) Pighini, Biochem. Zeitschr. 113, 231. - ${ }^{33}$ ) Pötzel, Jahrb. f. Psych. 31. - ${ }^{34}$ ) Rosenthal, St., Nissls Beitr. H. 2. - ${ }^{35}$ ) S piel me yer, Zeitschr. f. d. ges. Neurol. u. Psychiatr. 57. - ${ }^{36}$ ) Stern, Arch. f. d. ges. Psychol. 54. 37) Strü m pell, Dtsch. Zeitschr. f. Nervenheilk. 14, 50, 54. - ${ }^{38}$ ) Tho malla, Zeitschr. f. d. ges. Neurol. u. Psychiatr., Orig. 41. - ${ }^{39}$ ) Vogt, C. u. O., Journ. f. Psychol. u. Neurol. 18 u. 24. Vog t, C. u. C. Zeitschr. f. d. ges. Neurol u. Psychiatr., Ref. u. Erg. 21. $-{ }^{40}$ ) Wehi lin, Journ. f. Psychol. u. Neurol. 4. - ${ }^{41}$ ) Weil, A., Zeitschr. f. d. ges. Neurol. u. Psychiatr. 55. - ${ }^{42}$ ) Westphal, A., Arch. f. d. ges. Psychol. 60. - ${ }^{43}$ ) Westphal, C., Arch. f. d. Psych. 14. - ${ }^{44}$ ) Wilson, Brain 1912 und in Lewandowskys Handb. d. Neurol. 5. 